\title{
Development of Bio-Based Chitosan Films with Incorporated Chestnut Extract
}

\author{
Marijan Bajić, Uroš Novak, Blaž Likozar \\ Department of Catalysis and Chemical Reaction Engineering, National Institute of Chemistry \\ Hajdrihova 19, 1000 Ljubljana, Slovenia \\ marijan.bajic@ki.si; uros.novak@ki.si; blaz.likozar@ki.si
}

\begin{abstract}
Hydrolysable tannins have prominent biological activity and thus their industrial application is gaining importance in many fields. This study explored the possibility for the utilization of a commercially available chestnut extract (CE) as an active component in chitosan-based films intended for food packaging. Therefore, a set of chitosan-based films with incorporated CE was prepared and evaluated regarding physicochemical properties. The estimated total phenolic content (TPC) has revealed a maximal value of 19.5 mg ${ }_{\mathrm{GAE}}$ $\mathrm{g}_{\text {film }}{ }^{-1}$. Moreover, the moisture content $(M C)$ in the films has decreased (from $29.6 \%$ to $18.6 \%$ ), while tensile strength $(T S)$ has increased (from 13.5 MPa to 48.5 MPa) after the incorporation of CE. The observed coherence between TPC and evaluated properties has been confirmed by the existence of strong negative and positive linear correlations in the case of $M C$ and $T S$, respectively. In large, CE extract was found to be a promising candidate as an active component in chitosan-based films.
\end{abstract}

Keywords: Chitosan; Chestnut extract; Total phenolic content; Moisture content; Tensile strength; Food packaging.

\section{Introduction}

In the contemporary society, plastic waste is a serious environmental burden that presents a planetary boundary threat [1]. Bearing in mind the fact that single-use food packagings are one of the biggest sources of plastic waste, additional efforts should strive towards the utilization of alternative food packaging systems such as polymer-based films/coatings. Chitosan, a partly deacetylated derivative of biomass-based polymer chitin, is a prominent candidate for the preparation of a broad palette of eco-friendly materials, including those intended for food packagings [2].

Chitosan is a biocompatible, biodegradable, and non-toxic biopolymer whose characteristics highly depend on its degree of deacetylation and molecular weight [2]. It is endowed by a good film-forming capacity that makes chitosan one of the most sought biopolymers for the preparation of environment-friendly films for food protection [2]. The films' antioxidant and/or antimicrobial properties could be significantly boosted by the incorporation of various plant-based active components, thus helping in the extension of the shelf life of perishable foods [2,3]. The most recent studies have attempted the utilization of extracts/essential oils stemming from different parts of apricot (Prunus armeniaca) [3], mango (Mangifera indica) [4], hop (Humulus lupulus) [5], etc.

European chestnut (Castanea sativa) represents very important three in agro and forestry economy [6]. Industrial tannin extracts obtained from the chestnut wood have prominent antioxidant activity [6], and as such are interesting candidates as active components in chitosan-based films. Thus, the aim of the study was to explore the possibility to use CE as an active component in chitosan-based films. The films were evaluated in terms of $T P C$ as well as its correlation with $M C$ and $T S$.

\section{Material and Methods}

\subsection{Material}

High molecular weight chitosan (310-375 kDa; $\geq 75 \%$ deacetylated), lactic acid (purity $\geq 85 \%$ ), Folin-Ciocalteu's phenol reagent, and gallic acid were purchased from Sigma-Aldrich (Steinheim, Germany), while glycerol was from Pharmachem Sušnik (Ljubljana, Slovenia). CE (CasTan) was from Tanin Sevnica (Sevnica, Slovenia). Milli-Q ${ }^{\circledR}$ water was used throughout all experiments. 


\subsection{Preparation of chitosan-based films}

Chitosan was dissolved in $1.0 \%(\mathrm{v} / \mathrm{v})$ aqueous solution of lactic acid to reach the final concentration of $1.5 \%(\mathrm{w} / \mathrm{v})$, and glycerol was added as a plasticizer in the concentration of $30 \% \mathrm{w} / \mathrm{w}$ (based on the mass of chitosan) [5]. The mixture was stirred overnight at $1000 \mathrm{rpm}$ and $24{ }^{\circ} \mathrm{C}$, and vacuum-filtered through two layers of medical gauze. The addition of CE powder $[0.0 \%$ (control), $0.1 \%, 0.5 \%$, and $1 \%(\mathrm{w} / \mathrm{v})]$ was followed by 2 min long homogenization on Ultra-Turrax ${ }^{\circledR}$ T50 (IKA, Staufen, Germany) at $6000 \mathrm{rpm}$. Prepared film-forming solutions (FFS) were then left overnight to get rid of the bubbles and to stabilize the foam. Finally, the sticky foam and remaining bubbles were carefully removed from the mixtures using a laboratory spatula. The FFS were cast in polyurethane Petri dishes $\left(\sim 0.32 \mathrm{~mL} \mathrm{~cm}^{-2}\right)$ and dried at $40{ }^{\circ} \mathrm{C}$ for $48 \mathrm{~h}$. Obtained films were labelled based on the CE concentration in the FFS as CE_0.0 (control), CE_0.1, CE_0.5, and CE_1.0. The films' thicknesses were measured by a digital thickness gauge (Mitutoyo, Aurora, USA).

\subsection{Total phenolic content}

The TPC value of chitosan-based films was estimated using Folin-Ciocalteu's (FC) phenol reagent according to the protocol described elsewhere in the literature [5]. The results were expressed as the mass of gallic acid equivalent (GAE) per mass of the film.

\subsection{Moisture content}

$M C$ in chitosan-based films was determined by oven-drying of rectangular film samples $\left(\sim 1 \mathrm{~cm}^{2}\right)$ with a known mass at $105{ }^{\circ} \mathrm{C}$ for $24 \mathrm{~h} . M C(\%)$ was calculated from the difference between initial and dry mass of the samples [5].

\subsection{Tensile strength}

TS was determined by testing rectangular film samples $(8 \mathrm{~cm} \times 2 \mathrm{~cm}$; gage length segment $6 \mathrm{~cm})$ on the XLW Auto Tensile Tester (Labthink ${ }^{\circledR}$ Instruments, Jinan, China) equipped with a $100 \mathrm{~N}$ load cell, at a crosshead speed of 25 $\mathrm{mm} \mathrm{min}^{-1}$. It was calculated by dividing the load with the average original cross-sectional area in the sample gage length segment.

\subsection{Statistical analysis}

Statistical analysis was performed using the one-way ANOVA $(p<0.05)$ followed by Tukey's HSD post-hoc test, whereby different letters in the graphs indicate significantly different mean values. All experiments were done in triplicates and the results were expressed as the mean \pm standard deviation.

\section{Results and discussion}

\subsection{Preparation of chitosan-based films}

Obtained films were robust, stable, compact and easily-peeled off from Petri dishes. The films had a pale-brown to a dark-brown shade that was increasing in intensity along with increasing the amount of $\mathrm{CE}$. The thicknesses of the samples were $86 \pm 1 \mu \mathrm{m}, 67 \pm 2 \mu \mathrm{m}, 78 \pm 1 \mu \mathrm{m}$, and $84 \pm 1 \mu \mathrm{m}$ for CE_0.0, CE_0.1, CE_0.5, and CE_1.0, respectively.

\subsection{Total phenolic content}

The $T P C$ value is of high importance due to its relation to the antioxidant activity as well as other physicochemical properties of the films. The results of a crude estimation of TPC are depicted in Fig. 1. 


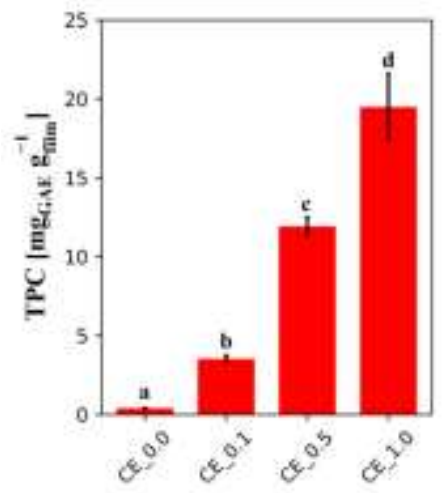

Fig. 1: Total phenolic content in chitosan-based films.

The TPC value was gradually increasing with the increase of $\mathrm{CE}$ concentration in chitosan-based films (Fig. 1). Accordingly, TPC in CE_0.0 was negligibly small (only $0.4 \mathrm{mg}_{\mathrm{GAE}} \mathrm{g}_{\text {film }}{ }^{-1}$ ), presumably due to the reaction between the FC reagent and $-\mathrm{NH}_{2}$ functional groups from chitosan [5 and ref. therein]. In the blended films, TPC was $3.5 \mathrm{mg}_{\mathrm{GAE}} \mathrm{g}_{\text {film }}{ }^{-1}, 11.9$ $\mathrm{mg}_{\mathrm{GAE}} \mathrm{g}_{\text {film }}{ }^{-1}$, and $19.5 \mathrm{mg}_{\mathrm{GAE}} \mathrm{g}_{\text {film }}{ }^{-1}$ for CE_0.1, CE_0.5, and CE_1.0, respectively (Fig. 1). By comparison, analogous chitosan-based films containing a supercritical $\mathrm{CO}_{2}$ hop extract had TPC of $1.5 \mathrm{mg}_{\mathrm{GAE}} \mathrm{g}_{\text {film }}{ }^{-1}, 7.0 \mathrm{mg}_{\mathrm{GAE}} \mathrm{g}_{\text {film }}{ }^{-1}$, and 10.5 $\mathrm{mg}_{\mathrm{GAE}} \mathrm{g}_{\text {film }}{ }^{-1}[5]$.

\subsection{Moisture content}

$M C$ content significantly affects the film performances [5]. Therefore, this parameter was determined by the gravimetric method and the results are summarized in Fig. 2.
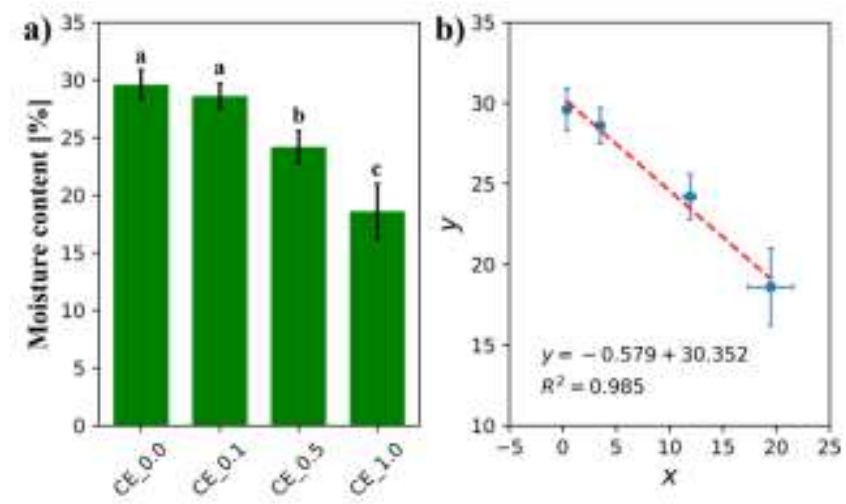

Fig. 2: Moisture content in chitosan-based films: a) moisture content; b) correlation between TPC (x-axis) and $M C$ (y-axis).

As compared to the control film, the values of $M C$ have decreased after the incorporation of CE (Fig. 2a). The control sample (CE_0.0) had 29.6\% of water, while other three samples had 28.6\% (CE_0.1), 24.2\% (CE_0.5), and 18.6\% (CE_1.0). The increased hydrophobicity of chitosan-based films was assumed to be due to intermolecular interactions between chitosan and incorporated tannins that prevent the establishment of chitosan-water hydrogen bonds [5]. This assumption is reinforced by the existence of a strong negative correlation between $T P C$ and $M C$ (Fig. 2b).

\subsection{Tensile strength}

Sufficient strength of the film material is crucial in providing mechanical integrity during transport and storage of food. The results of the films' TS are presented in Fig. 3. 

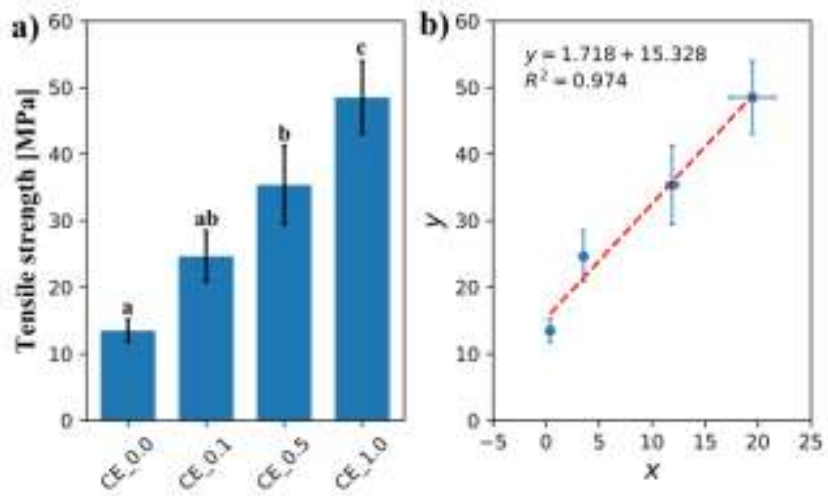

Fig. 3: Tensile strength of chitosan-based films: a) tensile strength; b) correlation between TPC (x-axis) and TS (y-axis).

The evaluated films have revealed TS from 13.5 MPa (CE_0.0) to 48.5 MPa (CE_1.0) (Fig. 3a). The presence of $\mathrm{CE}$ has heightened the films' resistance, in all probability due to the interactions between incorporated tannins and chitosan. This was additionally confirmed by the existence of a strong positive correlation between TPC and TS (Fig. $3 b)$. For instance, the presence of a hop extract in chitosan-based films has diminished their resistance, most likely due to a lower number of $-\mathrm{OH}$ groups in the hop's $\alpha$-acids and $\beta$-acids that can interfere with chitosan [5], as compared to tannins from CE. Thus, $\mathrm{CE}$ is supposed to acts as a cross-linker.

\section{Conclusion}

A possibility for the utilization of $\mathrm{CE}$ as the active ingredient in chitosan-based films has been successfully tested. The film with the highest tested concentration of CE had $M C$ of $18.6 \%$ and $T S$ of $48.5 \mathrm{MPa}$. It has been clear from the correlations between TPC and MC (negative correlation) as well as between TPC and TS (positive correlation) that films' physicochemical properties can be tailored by adding a proper amount of CE.

\section{Acknowledgements}

This work was financed by the BioApp project (Interreg V-A Italy-Slovenia 2014-2020). Ana Oberlintner is highly acknowledged for her help during the experimental work. CE was kindly donated by the company Tanin Sevnica (Sevnica, Slovenia).

\section{References}

[1] P. Villarrubia-Gómez, S. E. Cornell, J. Fabres, "Marine plastic pollution as a planetary boundary threat - The drifting piece in the sustainability puzzle," Mar. Policy, vol. 96, pp. 213-220, 2018.

[2] H. Wang, J. Qian, F. Ding, "Emerging chitosan-based films for food packaging applications," J. Agric. Food Che., vol. 66, no. 2, pp. 395-413, 2018.

[3] R. Priyadarshi, Sauraj, B. Kumar, F. Deeba, A. Kulshreshtha, Y. S. Negi, "Chitosan films incorporated with Apricot (Prunus armeniaca) kernel essential oil as active food packaging material," Food Hydrocolloid., vol. 85, pp. 158-166, 2018.

[4] K. Rambabu, G. Bharath, F. Banat, P. L. Show, H. H. Cocoletzi, "Mango leaf extract incorporated chitosan antioxidant film for active food packaging," Int. J. Biol. Macromol., vol. 126, pp. 1234-1243, 2019.

[5] M. Bajić, H. Jalšovec, A. Travan, U. Novak, B. Likozar, "Chitosan-based films with incorporated supercritical $\mathrm{CO}_{2}$ hop extract: Structural, physicochemical, and antibacterial properties," Carbohyd. Polym., vol. 219, pp. 261-268, 2019.

[6] G. Squillaci, F. Apone, L. M. Sena, A. Carola, A. Tito, M. Bimonte, A. De Lucia, G. Colucci, F. La Cara, A. Morana, "Chestnut (Castanea sativa Mill.) industrial wastes as a valued bioresource for the production of active ingredients," Process Biochem., vol. 64, pp. 228-236, 2018. 